\title{
Myocardial perfusion reserve by using CZT: It's a long way to the top if you wanna standardize
}

\author{
Emilia Zampella, MD, PhD, ${ }^{\mathrm{a}}$ Roberta Assante, $\mathrm{MD}$, PhD, ${ }^{\mathrm{a}}$ Valeria Gaudieri, MD, \\ $\mathrm{PhD},{ }^{\mathrm{a}}$ Carmela Nappi, MD, PhD, ${ }^{\mathrm{a}}$ Wanda Acampa, MD, PhD, ${ }^{\mathrm{a}, \mathrm{b}}$ and \\ Alberto Cuocolo, $\mathrm{MD}^{\mathrm{a}}$ \\ a Department of Advanced Biomedical Sciences, University "Federico Il", Naples, Italy \\ b Institute of Biostructure and Bioimaging, National Council of Research, Naples, Italy
}

Received Jul 2, 2019; accepted Jul 2, 2019

doi: $10.1007 / \mathrm{s} 12350-019-01817-6$

\section{See related article, pp. 876-884}

Myocardial perfusion imaging (MPI) using singlephoton emission computed tomography (SPECT) has long been the most common non-invasive test for management of patients with known or suspected coronary artery disease (CAD). The diagnostic and prognostic applications of SPECT MPI are traditionally based on visual and semi-quantitative assessment of the extent and severity of perfusion abnormalities, besides functional variables. ${ }^{1}$ However, standard semi-quantitative evaluation of myocardial perfusion is unable to assess subtle and/or diffuse alterations of myocardial blood flow (MBF) regulation. Myocardial flow reserve (MFR), obtained by the ratio between hyperemic and baseline MBF, is a well-validated index for the assessment of blood flow impairment, integrating the hemodynamic effects of epicardial coronary stenosis, diffuse atherosclerosis, and microvascular dysfunction. $.^{2-4} \mathrm{Car}-$ diac position emission tomography (PET)/computed tomography (CT) imaging allows absolute quantification of MBF and MFR over a wide range of perfusion tracers in animal models and humans. A preserved MFR is associated with low probability of coronary atherosclerosis and low risk of cardiac events; on the contrary, a reduced MFR is a significant marker that can help in stratifying CAD risk. ${ }^{5-8}$ However, its diffuse application

Reprint requests: Alberto Cuocolo, MD, Department of Advanced Biomedical Sciences, University "Federico II", Via Pansini 5, 80131 Naples, Italy; cuocolo@unina.it

J Nucl Cardiol 2021;28:885-7.

$1071-3581 / \$ 34.00$

Copyright (C) 2019 American Society of Nuclear Cardiology. in the clinical scenario is limited by the technical complexity and elevated operative costs. ${ }^{9}$ Previous studies investigated the feasibility of MFR quantification using dynamic scintigraphy and ${ }^{99 \mathrm{~m}} \mathrm{Tc}$ tracers in humans. ${ }^{10-14}$ Nevertheless, the dynamic collection of tomographic data, necessary to obtain MBF and MFR accurate measurements, is limited using conventional SPECT systems. The poor temporal resolution of conventional scintillation crystals does not allow to collect an adequate number of counts during changes in radiotracer concentration. In addition, varying angular projections and the slow rotation of the gantry limit the collection of dynamic data. Recent advances in SPECT technology, related to the introduction of newly dedicated cardiac cameras equipped with cadmium-zinc-telluride (CZT) detectors and software-based resolution recovery, might overcome most of the limitation of traditional systems. In CZT cameras, spatial and temporal resolution are greatly improved by performance of detectors and count density, respectively. ${ }^{15}$ Ben-Heim et al ${ }^{16}$ first evaluated the feasibility of dynamic tomographic imaging for quantification of regional and global MFR in humans, using a CZT camera (D-SPECT, Spectrum Dynamics, Israel). Ninety-five consecutive patients were evaluated by dynamic CZT SPECT imaging with ${ }^{99 \mathrm{~m}} \mathrm{Tc}$-sestamibi at rest and at peak vasodilator stress. The dynamic images were reconstructed, and a 2-compartment kinetic model was applied to calculate global and regional MFR index. Global MFR index was found to be higher in patients with normal imaging results than in those with abnormal MPI. Moreover, in 16 patients undergoing invasive coronary angiography, MFR index was also found to be lower in vascular territories supplied by obstructed arteries, showing a stepwise reduction with increasing extent of obstructive CAD. ${ }^{16}$

In the current issue of Journal of Nuclear Cardiology, de Souza et $_{a l} \mathrm{l}^{17}$ aimed to evaluate the feasibility of 
MBF and MFR absolute quantification, using a fast acquisition protocol in a commercially available CZT camera (Discovery 530, GE Healthcare, Milwaukee, USA). The authors also evaluated whether absolute measurements of MBF and MFR are related to perfusion imaging and angiographic severity of CAD. For this purpose, 41 patients with known or suspected CAD referred for coronary angiography, underwent a 1-day rest/stress dynamic imaging protocol using a CZT camera. Rest and stress dynamic phases were acquired for 11 minutes. Different from previous reports, ${ }^{18,19}$ routine-gated rest and stress perfusion images were obtained immediately after dynamic acquisition. At coronary angiography, CAD prognostic index was calculated in order to identify patients at high risk of disease. As expected, the authors observed a significant reduction in both stress MBF and global MFR in patients with abnormal SPECT scan, as compared to those with normal MPI results. In particular, MFR significantly decreases with increasing severity of the perfusion defect. According to angiographic findings, MFR appears to be inversely related to CAD prognostic index, showing a strength relationship between anatomical and perfusion measurements. ${ }^{17}$

Several important issues presented in the study by Souza et $a l^{17}$ deserve to be emphasized. The introduction of CZT detectors is considered a revolutionary milestone in technical innovation for nuclear MPI, offering a variety of interesting new possibilities. The increased sensitivity has led to a significant reduction in acquisition times, improving patients' comfort and optimizing the daily activity in nuclear cardiology laboratories. However, CZT technology is more sensitive to motion artifacts and any patient motion will simultaneously affect all the detectors, with a higher negative impact on image quality than during the longer acquisition with conventional SPECT. ${ }^{20}$ In previous reports, ${ }^{18,19}$ gated acquisition was performed up to 30 minutes or not included into imaging protocol, partially nullifying the advantages of the method with longer acquisition times. Differently, in the current study, the authors proposed a routine-gated rest perfusion images obtained immediately after dynamic acquisition, leading a shortened imaging protocol. Against this background, in order to take full advantage of CZT technology, a shortened imaging protocol is very gainful to promote the spread of the method into the clinical practice.

Another consideration arises from the evidence that the strength of MFR is the ability to help in overcoming the limitations of semi-quantitative MPI, in particular, in the identification of CAD. An important question to answer is if there is a physiologic threshold of hyperemic MBF or MFR that can be routinely used to accurately predict the presence of stenosis at coronary angiography. This issue has been largely investigated using PET/CT imaging; ${ }^{21}$ however, robust data on the CZT method are still lacking. Previous reports investigated the relationship between anatomical disease and perfusion measurements by CZT SPECT imaging, providing different results. Recently Zavadovsky et $a l^{22}$ aimed to determine the diagnostic power of MBF evaluation on dynamic CZT imaging in 23 intermediate risk patients in comparison with invasive coronary angiography and fractional flow reserve (FFR). By using 1-day dynamic rest-stress ${ }^{99 \mathrm{~m}} \mathrm{Tc}$-sestamibi perfusion imaging, stress MBF, MFR, and flow difference (obtained as the difference between stress MBF and rest MBF) were estimated. FFR abnormalities were best predicted by absolute stress MBF, MFR, and flow difference with values of $<0.54 \mathrm{~mL} / \mathrm{min} / \mathrm{g}$ (sensitivity $61.5 \%$ and specificity 93.3\%), $<1.48$ (sensitivity $69.2 \%$ and specificity $93.3 \%$ ), and $<0.18 \mathrm{~mL} / \mathrm{min} / \mathrm{g}$ (sensitivity $69.2 \%$ and specificity 100\%), respectively. Ben Bouallègue et $a l^{19}$ evaluated 23 patients with known multivessel CAD. The authors compared regional MFR, expressed as flow ratio (stress MBF/rest MBF) as well as flow difference with angiographic findings. Regional MFR indices were significantly lower in obstructed than in non-obstructed vessels. In particular, using a cut-off of 2, flow ratio was a good predictor of vessel obstruction with a sensitivity, specificity, and accuracy of $80 \%$, $85 \%$, and $81 \%$, respectively. Miyagawa $e t a l^{23}$ also proposed to validate MFR quantification by CZT camera in patients with multivessel CAD. Using a cut-off value of 1.3 for global MFR index, the sensitivity and specificity for diagnosing multivessel disease was $93.3 \%$ and $75.9 \%$, respectively. Differently, de Souza et $a l^{17}$ identified a global MFR cut-off point of 2.08 (with a sensitivity of $66.7 \%$ and a specificity of $84.6 \%$ ) for the diagnosis of high-risk CAD. Interestingly, the authors performed also a per vessel analysis in order to directly assess the relationship between coronary anatomy and regional MBF. They found that a regional MFR cut-off point of 2.2 provided a sensitivity of $63.2 \%$ and specificity of $74.1 \%$ in identifying an obstructive lesion in the corresponding artery. ${ }^{17}$

In the clinical practice, different severity thresholds for MFR may be considered, according to the wide variability related to subclinical and clinical condition, different radionuclides, scanners, imaging protocols, stress agents, and flow models. It has now been established that dynamic acquisition and quantification with CZT technology are feasible, but more data with larger population and multicenter studies are needed to identify global and regional specific cut-off values, for diagnostic and prognostic purposes. 


\section{Disclosure}

E. Zampella, R. Assante, V. Gaudieri, C. Nappi, W. Acampa, and A. Cuocolo declare that they have no conflict of interest.

\section{References}

1. Iskandrian AE, Hage FG, Shaw LJ, Mahmarian JJ, Berman DS. Serial myocardial perfusion imaging: Defining a significant change and targeting management decisions. JACC Cardiovasc Imaging. 2014;7:79-96.

2. Assante R, Zampella E, Arumugam P, Acampa W, Imbriaco M, Tout D, et al. Quantitative relationship between coronary artery calcium and myocardial blood flow by hybrid rubidium-82 PET/ CT imaging in patients with suspected coronary artery disease. J Nucl Cardiol 2017;24:494-501.

3. Gaudieri V, Acampa W, Rozza F, Nappi C, Zampella E, Assante $\mathrm{R}$, et al. Coronary vascular function in patients with resistant hypertension and normal myocardial perfusion: A propensity score analysis. Eur Heart J Cardiovasc Imaging. 2019. https://doi.org/10. 1093/ehjci/jez025.

4. Assante R, Acampa W, Zampella E, Arumugam P, Nappi C, Gaudieri V, et al. Coronary atherosclerotic burden vs. coronary vascular function in diabetic and nondiabetic patients with normal myocardial perfusion: A propensity score analysis. Eur J Nucl Med Mol Imaging 2017;44:1129-35.

5. Naya M, Murthy VL, Taqueti VR, Foster CR, Klein J, Garber M, et al. Preserved coronary flow reserve effectively excludes highrisk coronary artery disease on angiography. J Nucl Med 2014;55:248-55.

6. Zampella E, Acampa W, Assante R, Nappi C, Gaudieri V, Mainolfi CG, et al. Combined evaluation of regional coronary artery calcium and myocardial perfusion by (82)Rb PET/CT in the identification of obstructive coronary artery disease. Eur J Nucl Med Mol Imaging 2018;45:521-9.

7. Murthy VL, Naya M, Foster CR, Hainer J, Gaber M, Di Carli G, et al. Improved cardiac risk assessment with non-invasive measures of coronary flow reserve. Circulation 2011;124:2215-24.

8. Assante R, Acampa W, Zampella E, Arumugam P, Nappi C, Gaudieri V, et al. Prognostic value of atherosclerotic burden and coronary vascular function in patients with suspected coronary artery disease. Eur J Nucl Med Mol Imaging 2017;44:2290-8.

9. Ziadi MC. Myocardial flow reserve (MFR) with positron emission tomography (PET)/computed tomography (CT): Clinical impact in diagnosis and prognosis. Cardiovasc Diagn Ther 2017;7:206-18.

10. Storto G, Cirillo P, Vicario ML, Pellegrino T, Sorrentino AR, Petretta M, et al. Estimation of coronary flow reserve by Tc- $99 \mathrm{~m}$ sestamibi imaging in patients with coronary artery disease: Comparison with the results of intracoronary Doppler technique. J Nucl Cardiol 2004;11:682-8.

11. Pellegrino T, Storto G, Filardi PP, Sorrentino AR, Silvestro A, Petretta M, et al. Relationship between brachial artery flow-mediated dilation and coronary flow reserve in patients with peripheral artery disease. J Nucl Med 2005;46:1997-2002.
12. Petretta M, Soricelli A, Storto G, Cuocolo A. Assessment of coronary flow reserve using single photon emission computed tomography with technetium $99 \mathrm{~m}$-labeled tracers. J Nucl Cardiol 2008;15:456-65.

13. Daniele S, Nappi C, Acampa W, Storto G, Pellegrino T, Ricci F, et al. Incremental prognostic value of coronary flow reserve assessed with single-photon emission computed tomography. J Nucl Cardiol 2011;18:612-9.

14. Petretta M, Storto G, Pellegrino T, Bonaduce D, Cuocolo A. Quantitative assessment of myocardial blood flow with SPECT. Prog Cardiovasc Dis 2015;57:607-14.

15. Mannarino T, Assante R, Ricciardi C, Zampella E, Nappi C, Gaudieri V, et al. Head-to-head comparison of diagnostic accuracy of stress-only myocardial perfusion imaging with conventional and cadmium-zinc telluride single-photon emission computed tomography in women with suspected coronary artery disease. J Nucl Cardiol 2019. https://doi.org/10.1007/s12350-019-01789-7.

16. Ben-Haim S, Murthy VL, Breault C, Allie R, Sitek A, Roth N, et al. Quantification of myocardial perfusion reserve using dynamic spect imaging in humans: A feasibility study. J Nucl Med 2013;54:873-9.

17. de Souza ACDAH, Gonçalves BKD, Tedeschi AL, Lima RSL. Quantification of myocardial flow reserve using a gamma camera with solid-state cadmium-zinc-telluride detectors: Relation to angiographic coronary artery disease. J Nucl Cardiol 2019. http s://doi.org/10.1007/s12350-019-01775-z.

18. Agostini D, Agostini D, Roule V, Nganoa C, Roth N, Baavour R, Parienti JJ, et al. First validation of myocardial flow reserve assessed by dynamic (99m)Tc-sestamibi CZT-SPECT camera: Head to head comparison with (15)O-water PET and fractional flow reserve in patients with suspected coronary artery disease. The WATERDAY study. Eur J Nucl Med Mol Imaging 2018;45:1079-190.

19. Ben Bouallègue F, Roubille F, Lattuca B, Cung TT, Macia JC, Gervasoni $\mathrm{R}$, et al. SPECT myocardial perfusion reserve in patients with multivessel coronary disease: Correlation with angiographic findings and invasive fractional flow reserve measurements. J Nucl Med 2015;56:1712-7.

20. Benz DC, Buechel RR. Moving ahead with CZT technology. J Nucl Cardiol 2016;23:527-8.

21. Gould KL, Johnson NP, Bateman TM, Beanlands RS, Bengel FM, Bober R, et al. Anatomic versus physiologic assessment of coronary artery disease. Role of coronary flow reserve, fractional flow reserve, and positron emission tomography imaging in revascularization decision-making. J Am Coll Cardiol 2013;62:1639-53.

22. Zavadovsky KV, Mochula AV, Boshchenko AA, Vrublevsky AV, Baev AE, Krylov AL, et al. Absolute myocardial blood flows derived by dynamic CZT scan vs invasive fractional flow reserve: Correlation and accuracy. J Nucl Cardiol 2019. https://doi.org/10. 1007/s12350-019-01678-z.

23. Miyagawa M, Nishiyama Y, Uetani T, Ogimoto A, Ikeda S, Ishimura $\mathrm{H}$, et al. Estimation of myocardial flow reserve utilizing an ultrafast cardiac SPECT: Comparison with coronary angiography, fractional flow reserve, and the SYNTAX score. Int J Cardiol 2017;244:347-53.

Publisher's Note Springer Nature remains neutral with regard to jurisdictional claims in published maps and institutional affiliations. 